\title{
EL ESTADO Y SU FRACTURA CON LA EDUCACIÓN SUPERIOR
}

\author{
Juan Guillermo Licona Piña *
}

\section{RESUMEN}

La redefinición de las relaciones entre el gobierno y la educación superior, en el marco de la globalización, evidencian un adelgazamiento en la responsabilidad de quienes diseñan e instrumentan las políticas educativas para la universidad. El discurso oficial no acaba por traducirse en hechos concretos en beneficio de los que aspiran, asisten y egresan de dichas instituciones.

Una mirada profunda, reflexiva y crítica permite apreciar "una fractura". Un cierto tipo de fisura, que sin saber precisamente en que grado o nivel se padece, sí requiere urgente atención de parte de los que directa o indirectamente tienen que ver con los estudios universitarios.

Quienes aspiramos a una sociedad más humana no podemos seguir dejar pasando, como si no pasara nada, tenemos el compromiso ineludible de actuar personal y colectivamente en estrategias para diluir el efecto corrosivo del neoliberalismo.

\section{ABSTRACT}

The redefinition of relations between the government and higher education, in the context of globalization, betrays a dwindling responsibility from those who design and implement education policy for higher education. The official discourse has not translated into concrete actions in benefit of those who apply, attend and graduate from institutions of higher learning.

A profound, reflective and critical look reveals a "break" or fissure, while whithout exactly knowing to what degree or at what level, requires urgent attention on the part of those directly or indirectly responsible for higher education policy.

Those of us who to build a more humane society cannot allow this break to continue as if nothing is happening. We have the inalienable duty to act individually and collectively on strategies to dilute the corrosive effects of neo-liberalism

\author{
Lic. en Administración en la Universidad Autónoma de Hidalgo, Profesor en \\ la especialidad de Psicología Educativa por la Normal Superior de Hidalgo, \\ maestrante en educación: campo práctica educativa, en la UPN, Unidad \\ Pachuca y en Educación Superior por la Universidad la Salle de Pachuca \\ juanlicona3@yahoo.com.mx
}


Los actuales procesos sociales, culturales y políticos se han visto agobiados por las presiones que ejerce un modelo, que aun cuando no acaba por definirse estructuralmente, ha sido implacable en la erosión de las razones que han dado sentido y orientación a la evolución del género humano, en su individualidad y como colectivo social

El fenómeno que conocemos como globalización ha impactado de distintas formas y alcances a prácticamente todas las dimensiones de la actividad del hombre.

El campo educativo no ha escapado a su influencia. Por ahora, la tenebrosa sombra de la globalización oscurece los más altos ideales de la educación superior de México y el mundo. Guerra $(2001,1)$ sustenta que "la universidad está pasando por un periodo de crisis". Una crisis que para el caso de México se asocia a la falta de capacidad en atención a la creciente demanda que él mismo ha impulsado, a los procesos de exclusión de los estudiantes tanto en el ingreso como para su permanencia en el mismo, a la falta de correspondencia entre su oferta educativa y el mercado laboral, la cuestionada preparación de sus cuadros docentes y la pertinencia de sus contenidos curriculares, los insuficientes recursos y cada vez más recortados presupuestos que asigna el Estado para sus objetivos sustanciales, etc.

En las siguientes líneas reflexiono entorno a la situación que guarda la relación entre el Estado (como representante de los intereses nacionales) y la educación superior, mediada por la globalización. El título del trabajo va en el sentido de develar ciertas acciones en el ámbito educativo instruidas por el gobierno de México, desde los años noventa hasta nuestros días, que guardan una gran dosis de lo que en esencia se conoce como globalización, las que de alguna manera inciden en la pérdida creciente de atención y cuidado en el subsistema de educación superior, y aún más, esas acciones tienen en común favorecer los procesos de adecuación de la educación al neoliberalismo. Bajo esta óptica, Ibarra $(2003,2)$ señala "la renovada presencia del Estado como fuerza desde la que han venido operando la privatización, la comercialización y la desregulación de las entidades públicas", siendo las educativas parte de ellas.

Tengamos presente que el instrumento principal de toda política pública es su presupuesto y la educación es una clase de dicha política a la que paulatinamente le han asignado menores recursos económicos en términos relativos, lo que supone la postergación y/o reducción en los alcances de los objetivos e incluso el fracaso de proyectos educativos en el nivel superior por lo que implica la atención gubernamental.

La fractura, a la que alude el título de este ensayo, es entendida como la ruptura o separación parcial de dos entes sociales. Si bien, es cierto que en la 
historia de esta relación han existido distanciamientos de diversa índole y de alcances diferenciados, se había contado con la disposición y los medios para construir alternativas de solución a corto y mediano plazo. Sin embargo, la configuración de los actuales problemas es producto de las tensiones que desde hace varios años han venido operando bajo la lógica del economicismo.

La ruptura implica falta de coordinación, trae implícita una falta de comunicación que no sólo afecta al uno o al otro, implica desventajas para ambos, tanto para la universidad como para el Estado; y de esta malsana convivencia el problema se extiende a otros espacios de la sociedad que en forma directa o indirecta se manifiestan de variadas formas en la actividad humana.

Bien lo recuerda Sylvie Didou, (citada por Molano 2003, 7):

"En México educación superior solía percibirse como un programa social y político en sí mismo... se esperaba recibir algo en retorno en términos de desarrollo del país. Pero actualmente este enfoque sensible a la sociedad está dando paso a una actitud meramente comercial".

Es urgente retomar una de las prioridades del quehacer universitario y que fue garante en su estructura como institución privilegiada de la sociedad: la de convertirse en creadora y generadora de individuos con capacidad para transformar la realidad social en beneficio de sus integrantes. Así, su relevancia implica la posibilidad de gestionar y vitalizar las formas productivas e influir en torno al modelo económico que más convenga al país sin perder de vista su contexto sociopolítico y cultural.

Lamentablemente el eco de la globalización ha penetrado en la ideología gubernamental y ésta ha priorizado el crecimiento de los capitales. Con ello, la tarea principal de los centros universitarios se ha diluido ante las reorientaciones (presiones) que en materia de política educativa ha instruido el gobierno

El Estado trabajaba en la definición de políticas educativas sustentadas en los apremiantes requerimientos de los mexicanos. Las instituciones de educación superior se constituían en centros operativos, sin desconocer y ejerciendo su plena autonomía, para alcanzar los objetivos definidos en los proyectos nacionales con apego a su entorno. En este sentido, Mungaray $(2001,5)$ estipula que "gobiernos y la sociedad civil tienen que asumir un rol activo para combatir las nuevas y viejas desigualdades". Lo que el Estado hace y deja de hacer en materia educativa se traduce en un fuerte impacto en el quehacer de las instituciones de educación superior. La actividad pasiva, o la dinámica estéril, contrarias a los contenidos discursivos de los responsables en materia educativa.

El enfoque que sigo en el tratamiento de este documento adopta la postura de lo que Bourdieu $(2002,11)$ Ilama globalicrítico, es decir, "críticas a la globalización neoliberal como la identidad de proyecto". Es una protesta sustentada pero que 
busca conformar una propuesta con los aportes de ciertos pensadores e investigadores en este tema.

Para iniciar busquemos una explicación de lo que es la globalización e inmediatamente vincularla con la educación superior. Los encargados de descifrar, interpretar y explicar esta fenomenología: filósofos, sociólogos, educadores y demás integrantes que conforman las ciencias sociales y humanas, nos ofrecen ciertas aproximaciones a la noción de lo que implica dicho término.

Para Molano $(2003,2)$, es en esencia "la profundización de la dominación neocolonial centrada en el capital financiero especulativo internacional que ha puesto a su servicio el enorme desarrollo de las fuerzas productivas a partir de los desarrollos de la biotecnología, la robótica, las telecomunicaciones; generando una desproporcional socialización y mundialización de las relaciones económicas, sociales, políticas, culturales y militares entre la superpotencia de América, las otras y las naciones del tercer mundo". Destaquemos aquí el papel que juegan los avances científicos y tecnológicos subordinados a intereses puramente económicos y la preeminencia de ciertas áreas del conocimiento en detrimento de las sociales y humanísticas. En este sentido, Ibarra $(2003,6)$ dice:

"Las políticas y programas de reestructuración de la educación superior en diversos países del mundo, aunque distintos en su conformación local y su operación específica, confluyen bajo los imperativos paradigmáticos de la privatización, la desregulación y la competitividad".

También, dice Morea $(2002,1)$ que la globalización "es el conjunto de procesos que conducen a un mundo único". Y que además "las sociedades se vuelven interdependientes en todos los aspectos de su vida, política, económica y cultural, y el alcance de tales interdependencias deviene realmente global". Esta interpretación aparenta cierta mesura, algo de neutralidad. Sin embargo, la inclusión del término interdependencia no parece corresponder a nuestra experiencia. Interdependencia es entendida como una necesidad recíproca, en la que los beneficios son compartidos en términos equitativos al igual que las necesidades; por lo tanto no se puede creer en una interdependencia entre la política educativa y la dimensión cultural. Pensemos en los países que han sido orillados a ser en gran parte, dependientes de otros que son absolutamente independientes por lo que hace a la toma de decisiones soportadas en sus capacidades económicas e inventarios bélicos.

En el caso de Fernández $(2002,5)$ opina que "como categoría histórica la globalización es un equivalente a la internacionalización económica [...] Es un fenómeno vinculado con el desarrollo capitalista. Se trata de fenómenos de interacción política y no de leyes naturales de la economía o de la tecnología". Más adelante continúa: "desde la perspectiva de la sociología del conocimiento se le instala como un paradigma montado sobre varias falacias, mitos o slogan, como que es un fenómeno nuevo, como genio y homogeneizante que conduce a la democracia, el progreso y el bienestar universal; que acarrea la desaparición 
progresiva del estado". Estas precisiones aclaran las mutaciones propiciadas en la inconsciente generación de la riqueza y la sobreexplotación tecnológica, además de que desenmascara a los supuestos utilizados para su ascensión.

Si es falsa la tendencia a la democracia, el progreso y el bienestar universal, entonces dónde queda el papel que desempeñan las universidades. Acaso no es cierto que estas aspiraciones descansen en gran medida en los resultados que entregan al pueblo dichas instituciones. Ibarra $(2003,4)$, nos advierte "hoy se encuentra en debate si estas transformaciones suponen el desplazamiento de la universidad como referente cultural básico de la sociedad, para adquirir en adelante el estatuto menor que poseen las empresas que prestan algún servicio a la sociedad". Si esta apreciación es correcta, la universidad dejaría de ser "institución" de la sociedad para devenir tan sólo en organización de mercado.

Enseguida, veamos cómo son explicadas las formas en que la globalización se filtra hasta las instituciones de educación superior. En la radiografía de la globalización aparecen las corporaciones multinacionales cuyas matrices se ubican principalmente en Estados Unidos, en algunos centros de Europa y Japón. Desde ahí, los centros de poder deciden los flujos económicos con la visión de la máxima ganancia con el menor costo, y a costa de todo.

Ahora bien, las multinacionales o trasnacionales han necesitado la creación de instituciones que operen al interior del colectivo de los países cliente. La Organización Mundial del Comercio, El Banco Mundial, El Fondo Monetario Internacional entre otras. Ellas, las huestes de los poderosos, en las dos últimas décadas, de manera creciente han insistido en tal promoción de proyectos en la educación superior. Bajo el argumento de que los países requieren de apoyos extraordinarios, pues sus presupuestos educativos son precarios, inducen la oferta de atractivos empréstitos, siempre y cuando los gobiernos se conviertan en garantes de las imposiciones que les sean determinadas. La paulatina ingerencia no ha sido posible más que con la actitud, complaciente de los responsables del sistema educativo nacional.

La ingerencia que tienen las representaciones internacionales de financiamiento de las IES se han asumido en organismos de ordenamiento y vigilancia que definen las normas a través de las cuales se han de aplicar los presupuestos que proporcionan en calidad de préstamo. No existe neutralidad en las intenciones, lo que posibilita y acompaña a esos recursos está en función de las posibilidades en cuanto a la rentabilidad de los proyectos educativos. Paralelamente, se entiende que la ministración pública disminuye su grado de participación en el financiamiento educativo. Es decir, la intervención de esas agencias especializadas ha sido lo que justifica y posibilita las políticas austeras en materia educativa del nivel superior.

Sabemos que es responsabilidad del gobierno administrar y ordenar la asignación de recursos económicos por lo que hace a los financiamientos regulares, así como de aquellos cuya particularidad hace que se enfoquen a las 
prioridades educativas del país. La confianza que la sociedad deposita en sus representantes hace suponer que ellos tienen la visión y capacidad para traducir nuestras necesidades en oportunidades de desarrollo a través de amplios proyectos educativos.

Las reformas impulsadas en esta materia han sido aduladas por los contenidos discursivos. Es innegable la gran capacidad del gobierno para construir los referentes y argumentos que dan cuenta de una complicada realidad social. La construcción teórica que hacen logra articular causa-efecto que desde distintas ópticas explican la génesis de los problemas educativos. El problema ha sido la poca efectividad de las soluciones, la distancia entre esa capacidad de respuesta y la satisfacción de necesidades educativas cada vez es mayor.

Una institución que ha venido consolidándose es la Asociación Nacional de Universidades e Instituciones de Educación Superior (ANUIES). La nobleza de sus propósitos queda opacada cuando se constata que en determinadas decisiones ha legitimado los cursos de acción que ha determinado el Estado, las que a su vez han emanado de organismos internacionales o puramente empresariales.

Por un lado, sin que se considere un justificante que aminore la responsabilidad oficial, las continuas contradicciones internas en la política nacional entre los poderes de la unión han hecho que el ejecutivo recurra a pedir o aceptar, según sea el caso, el supuesto respaldo de las instituciones mundiales crediticias. También se tiene que, como resultado de las históricas resquebrajadas economías del país, parece no tener alternativa para aceptar incondicionalmente recursos monetarios externos.

En cualquier caso, es evidente la falta de capacidad de los gobiernos para encarar las demandas sociales del sector educativo. Guerra $(2001,1)$ indica que "la tendencia más perniciosa es la intervención del estado evaluador". En nuestro país se tienen evidencias claras de tal descuido. La privatización de la educación es un tema vigente en la agenda de los organismos internacionales y su intención parece concretarse ante el desliz político y económico del estado, por ejemplo: las facilidades otorgadas para el crecimiento desmedido de las escuelas particulares de educación superior sin que medie la menor vigilancia de su currículo, de sus métodos y procesos, de las instalaciones, así como de sus resultados. La colectiva presencia de franquicias extranjeras que prestan el servicio y que gozan de exenciones fiscales.

Para ninguno es desconocido que muchas de las más importantes decisiones que le competen al Estado están ahora en poder del mercado. No desconocemos que la educación condicionada por la oferta y la demanda refleja una tendencia significativamente creciente desde la óptica de la libertad de elegir. La esencia teórica de la globalización es que el mercado se imponga junto y paralelamente a la renuncia del Estado ante sus ocupaciones de carácter social. 
Si cuando la educación era exclusivamente guiada por los ideales revolucionarios, consagrados en el Artículo Tercero Constitucional, la distancia que mediaba entre las aspiraciones a la movilidad social y su concreción eran considerables, ahora aparecen y se quedan, en la mayoría de la juventud, en su delirio imaginario con sello de inalcanzable.

En el espectro de la globalización, la educación superior sólo es posible para unos cuantos, no aplica para el grueso de la sociedad. Tradicionalmente, el sistema es selectivo, lo ha sido desde hace tanto que parece haber penetrado el sentido común de la población. El gobierno es indiferente ante esta necesidad e imparcial en esta inequidad.

Los últimos reportes censales, indican que la cobertura en la educación superior no rebasa la cuarta parte de la población en edad escolar, si a esto le añadimos la población potencial que se irá sumando en los próximos años, el problema toma dimensiones alarmantes. Ibarra $(2003,8)$ : "la educación superior atiende en su conjunto a 1837,000 estudiantes, que corresponden a un poco mas del $17.7 \%$ de la población en edad de acceder a este nivel educativo".

Reconocer también, que quienes han sido objeto de atención educativa son criticados y cuestionados por los procesos deficientes en los que transita, y para aquellos que tienen las máximas notas, saben bien que no les representa ninguna garantía para ocuparse productivamente en el mercado profesional, un mercado altamente excluyente.

Tengamos presente que la orientación general, la cantidad y calidad de la educación que en los diversos niveles proporciona la sociedad es producto de estimaciones, de carácter político.

La Organización Mundial del Comercio (OMC) va concretando sus aspiraciones, la tendencia a convertir las escuelas en entidades de lucro, con la venta del servicio al cliente y paradójicamente la alienación del conocimiento y del capital humano, que despoja al sujeto de su esencia para convertirlo en objeto de los intereses capitalistas, desprovisto de la ética que en otrora se pregonaba o de la "ética planetaria" a la que hoy urge Lyotard $(1999,4)$. Opina Guerra $(2001,3)$ que " se está acentuando a nivel mundial el concepto de la universidad como proveedora de la mano y mente de obra para el sector productivo y las carreras orientadas a la filosofía y humanidades cada vez mas perdiendo su valor".

Lo anterior se puede apreciar al analizar las prioridades educativas del gobierno. Así tenemos que es el nivel superior el que más recortes presupuestarios ha experimentado. La reorientación de los recursos hacia la educación básica según las recomendaciones internacionales menguó la capacidad de la universidad en distintos rubros. Hoy, la incertidumbre financiera encarece la eficacia y eficiencia en la planeación educativa del tercer nivel. 
Por lo anterior, en las universidades se tienen pocos profesores de tiempo completo en comparación con los que asisten académicamente por horas.

Por otro lado, se prefiere seguir impulsando a nivel nacional y estatal la creación y desarrollo de los estudios enmarcados en las universidades tecnológicas para preparar o mejor dicho adiestrar y capacitar a técnicos superiores en un plazo de dos años, con sostenimiento público. Es la respuesta a la exigencia del desarrollo sustentado en el capitalismo.

Sería deshonesto asentar que no existen ventajas y beneficios de la economía de mercado, lo que preocupa es la política del dejar hacer y dejar pasar, como eje de la política educativa, que surge del gobierno, las metas en términos de justicia social y equidad podrían enterrarse en el olvido.

Es cierto, necesitamos como siempre, estudiantes brillantes con destacados conocimientos en la ciencia y tecnología. Hay coincidencia de que el progreso de México requiere de estudiantes y egresados innovadores; que al mismo tiempo sean palanca para llevar nuestros productos al extranjero y empujen las dinámicas comerciales de los estados, a través de centros productivos competitivos, con la consecuente generación de trabajos dignos y de un ingreso creciente y mejor distribuido. Esas cualidades serían insuficientes si no formamos ciudadanos cívicos y socialmente útiles.

Se tienen que repensar las IES, principalmente a las públicas con el propósito de concretar las alternativas educativas de la población real y potencial que demanda acceder a ellas; enaltecer la enseñanza y generar verdaderas oportunidades de desarrollo profesional y movilidad social para los egresados; así como tener profesionales bien formados y preparados en el campo de la ciencia, la tecnología y las humanidades.

En los últimos años se ha producido un crecimiento significativo de la matrícula en estos niveles; sin embargo, el rezago en la cobertura y en la calidad sigue siendo elevado. Es falsa la apreciación de que existe un número excesivo de alumnos en educación superior. El rezago educativo es evidencia de la perversa política que ha seguido el gobierno. Al respecto, Mungaray $(2001,4)$, hace la siguiente convocatoria "La impostergable necesidad de redefinir la función tradicional de las instituciones públicas sin excepción, constituyendo procesos de reingeniería organizacional que permitan tender puentes entre el conocimiento que se produce en los centros de investigación públicos y la sociedad civil, propiciando flujos de interacción recíproca entre el estado y la sociedad con el fin de promover el desarrollo económico y social de los pueblos".

Guerra $(2001,5)$ pone el énfasis en que "el aspecto más visible de la crisis ha sido financiamiento público que se ha venido contrayendo, provocando el incremento de la centralización en las autoridades educativas, dificultando así la innovación en las organizaciones académicas educativas". Además, cada sector 
que recibe financiamiento público debe competir por los fondos y comprobar que el uso que hace de los recursos sea el adecuado.

En este contexto se tiene una asfixia financiera que padecen nuestras universidades, institutos, centros de investigación científica y de cultura pública.

No pueden generarse condiciones como las que pretende promover el ejecutivo federal, de manera casi a ciegas de darle cumplimiento a los indicadores de la OCDE, del BM, del BID o del FMI. Ibarra, (2003) destaca el hecho de que "la consideración de las necesidades que le han impuesto los procesos de globalización y regionalización a las universidades, hacen que se encuentren hoy subsumidas a la economía y el mercado, perdiendo la autonomía de la que gozaron en otros momentos.

Rehacer la relación entre universidad y Estado en beneficio de nuestra sociedad es posible. Se requiere que los actores y representantes de dichas instituciones vuelvan su mirada hacia las condiciones en las que se encuentran miles de mexicanos. Al principio se mencionó que el modelo que delinea a la globalización no se ha concretado, quiere decir que existen espacios en donde cabe la posibilidad de actuar en bien de la humanidad a través de la educación, sin que por ello se renuncie las posibles ventajas de acceder a un mundo unificado por medio de la globalización.

\section{REFERENCIAS}

BOURDIEU, Pierre. Ni Globalifilicos, ni globalifóbicos, sino globalicríticos: Jaime Preciado Coronado. Most-junesco. Universidad Federal de Río Grande. Do Soul, Sest. Porto Alegre. 30 de enero 2002. http://www.unesco.org/most/wsf/preciado.pdf.

FERNÁNDEZ, Mauro. Globalización, poder y educación pública (1). http://www. unam.mx/ceiich/educación/Saxe.htm.

LYOTARD, Jean Francois. Ética, postmodernidad y globalización. Los pensamientos son nubes. http:/www.cess.org.ar/macro/43-jul99/0799doc3.htm.

GUERRA, Ernesto. La educación superior en el contexto mundial. http//ccu.mazuasnet.mx/maryarena/julio 01/tendenciasmundiales.htm Revista mar y arena. Consultada el 19 de mayo de 2005.

IBARRA, Eduardo (2003) Capitalismo académico y globalización: la universidad reinventada. http://www.geocities.com/ibarra-colado

MOLANO, Friday. La educación pública en el contexto de la globalización. Análisis de los efectos de las políticas de la globalización impulsadas por la OMC y 
el ALCA sobre la educación pública". Centro de medios independientes de Colombia. Mayo 16, 2003.

MOREA, Lucas. Proceso de la globalización y su impacto en la educación latinoamericana. http://www.monografías.com/trabajos13/procglo/procglo.shtml.

MUNGARAY, A. (2001) La educación superior y el mercado de trabajo profesional. Revista electrónica de investigación educativa, 3 (1) Consultado el día 23 de mayo de 2005 en http://redie.ens.uabc.mx/vol3no1/contenidopmungaray,html

* Lic. en Administración en la Universidad Autónoma de Hidalgo, Profesor en la especialidad de Psicología Educativa por la Normal Superior de Hidalgo, maestrante en educación: campo práctica educativa, en la UPN, Unidad Pachuca y en Educación Superior por la Universidad la Salle de Pachuca juanlicona3@yahoo.com.mx 